KA-THEP-1989-XX

Manusk. Fak. Math. u. Inf.

Mannheim Nr. 101.1989

\title{
Krichever-Novikov Algebras \\ for More than Two Pointe: \\ Explicit Generators
}

\author{
Martin Schlichenmaier
}

101.1989

July 89

Faculty of Mathematics

and Computer Science

University of Mannheim, A5

D-6800 Mannheim 1

Federal Republic of Germany

\footnotetext{
Abstract

For the Krichever-Novikov algebras and modules of meromorphic vector fields and forms with more than two poles on higher genus Riemann surfaces explicit expressions of the generators are given. These are expressions in terms of theta functions and prime forms for $g \geq 1$, expressions in terms of the Weierstras $\sigma$ function for $g=1$ and rational expressions for $g=0$.
} 


\section{Introduction}

In $[1,2]$ Krichever and Novikov studied the algebra of meromorphic vector fields which have poles only at two generic but fixed points on a Riemann surface of arbitrary genus $g$. This algebra (with or without) central extension is now usually called Krichever-Novikov algebra (short KN algebra). It is a generalization of the Virasoro algebra to higher genus and it is of considerable interest in conformal field theory $([6-8]$ and references [6-14] in [s]). In [s] I reported on work done in [4]. The above results where generalized to the situation where one allows the meromorphic vector fields and forms to have poles at more than two points. Similar results were achieved independently by Reiner Dick [s].

In [3] ( and [5]) the existence and uniqueness of a special set of generators and of a basis of these generalized $\mathrm{KN}$ algebras and modules were proven by Riemann-Roch type arguments. Due to special interest in more explicit forms of the basis which were used in [6-8] to calculate propagators in the two point case I decided to report in this supplement to [ 3 ] on the results [ 4 ] concerning these more explicit forms. This will be done for Riemann surfaces of genus $g \geq 1$ by the use of theta functions and prime forms. In the $g=1$ case I give an alternative description in terms of the Weierstrab $\sigma$ function. For the sake of completeness I repeat for $g=0$ the result in terms of rational functions, as it can already be found in $[3,5]$.

For the details of the notation I refer to [3]. Let me just repeat the fundamental definitions. We start with $X$ a Riemann surface of genus $g$ and choose a set of distinct points $P_{1}, P_{2}, \ldots, P_{k}(k \geq 2)$ which are in generic position. The $\mathrm{KN}$ algebra $\mathrm{KN}\left(P_{1}, P_{3}, \ldots, P_{k}\right)$ is the Lie algebra of meromorphic vector fields which are holomor. phic on $X \backslash\left\{P_{1}, P_{2}, \ldots, P_{k}\right\} . F^{\lambda}\left(P_{1}, P_{2}, \ldots, P_{k}\right)$ is the vector space of meromorphic forms of weight $\lambda \in \mathrm{Z}$ which are holomorphic on $X \backslash\left\{P_{1}, P_{2}, \ldots, P_{k}\right\}$. A meromorphic form of weight $\lambda$ is a meromorphic section of the line bundle $K^{\otimes \lambda}$. Here $K$ denotes the canonical line bundle. Its sections are the (1.) differentials. By taking the Lie derivative with respect to the vector fields in the $\mathrm{KN}$ algebra, $F^{\lambda}\left(P_{1}, P_{2}, \ldots, P_{k}\right)$ will become a Lie algebra module over $\mathrm{KN}\left(P_{1}, P_{2}, \ldots, P_{k}\right)$, called the $\mathrm{KN}$ module of weight $\lambda$. Of course, $F^{-1}\left(P_{1}, P_{2}, \ldots, P_{k}\right)=\mathrm{KN}\left(P_{1}, P_{2}, \ldots, P_{k}\right)$. One can also allow non-integer $\lambda$ by considering spin bundles (for half integer $\lambda$ ) or coverings of $X$ (for rational or real $\lambda$, see $[4,8])$.

We denote by

$$
f^{\lambda}\left(n_{1}, n_{2}, \ldots, n_{k}\right), \quad \text { all } n_{i} \in \mathbf{Z}
$$

a form of weight $\lambda$ which has a zero of order $n_{i}$ at $P_{i}$ for $i=1, \ldots, k$ and is holomorphic elsewhere. As usual a zero of negative order is a pole. We set

$$
M(\lambda)=(2 \lambda-1)(g-1)-1 \text {. }
$$


It was proven in [4] and reported in [3,Prop.1 and 2] that in the case $g=0$ or $g \geq 2$ and $\lambda \neq 0,1$ the elements (1) with $\sum_{i=1}^{k} n_{i}=M(\lambda)$ are uniquely determined (up to multiplication with a constant) and that the set of such elements forms a set of generators for $F^{\lambda}\left(P_{1}, P_{2}, \ldots, P_{k}\right)$. By choosing a local coordinate $z_{k}$ at $P_{k}$ and requiring that the lowest coefficient in the Laurent series expansion of $(1)$ at $P_{k}$ should be equal to 1 , we can fix (1) uniquely. In the remaining cases a finite set of generators have to be modified. A vector space basis of $F^{\lambda}\left(P_{1}, P_{2}, \ldots, P_{k}\right)$ can be gained as a subset of the above set of generators, see $[s]$ for details.

\section{The construction via theta functions and prime forms in the case}

\section{$g \geq 1$. General case.}

Here I follow the technique used in [6]. Let me first collect the relevant facts on the building blocks-[9-12],

Let $X$ be a Riemann surface of genus $g \geq 1$. $a_{1}, a_{2}, \ldots, a_{g}, b_{1}, b_{2}, \ldots, b_{g}$ a symplectic homology basis. $\omega_{1}, \omega_{2}, \ldots, \omega_{g}$ the corresponding set of holomorphic (1-)differentials

$$
\oint_{a_{i}}=\delta_{i, j}, \quad \oint_{b_{i}}=\pi_{i, j}, \quad \Pi=\left(\pi_{i, j}\right)
$$

The Jacobian $\operatorname{Jac}(X)$ of $X$ is given as

$$
\mathbb{C}^{g} / L, \quad L=\mathbf{z}^{g} \oplus \Pi \cdot \mathbf{z}^{g} .
$$

The theta function is defined as

$$
\vartheta(z, \Pi)=\sum_{n \in \mathbf{Z}^{g}} \exp \left(\pi \mathbf{i}^{t} n \cdot \Pi \cdot n+2 \pi \mathbf{i}^{t} n \cdot z\right)
$$

for $z \in \mathbb{C}^{g} . \vartheta$ is a holomorphic function on $\mathbb{C}^{g}$ with the following quasi-periodic behaviour under translation of $z$ with vectors from the lattice $L$ ( $m \in Z^{g}$ )

$$
\begin{aligned}
\vartheta(z+m, \Pi) & =\vartheta(z, \Pi) \\
\vartheta(z+\Pi \cdot m, \Pi) & =\exp \left(-\pi \mathbf{i}^{t} m \cdot \Pi \cdot m-2 \pi \mathbf{i}^{t} m \cdot z\right) \cdot \vartheta(z, \Pi) .
\end{aligned}
$$

We fix $X$ and our homology basis. Hence we will drop the period matrix $\Pi$ in the notation.

The Riemann surface can be embedded via the Jacobi map $J$ into its Jacobian. For this we choose a base point $Q \in X$ and set

$$
X \rightarrow \operatorname{Jac}(X), \quad P \mapsto J(P):=\left(\int_{Q}^{P} \omega_{1}, \int_{Q}^{P} \omega_{2}, \ldots, \int_{Q}^{P} \omega_{g}\right) \bmod L .
$$


$\left(\int_{Q}^{P}\right.$ is an arbitrary path from $Q$ to $P$.) I will use $J(P)$ for both the image of $P$ in $\operatorname{Jac}(X)$ and the multi valued image in $\mathbb{C}^{g}$.

The pullback $P \mapsto \vartheta(J(P)$ of $\theta$ is a multi valued function on $X$. Riemann's theorem [10,I,p.149]] says: There exists a vector $\Delta \in \mathbb{C}^{9}$ (the Riemann vector) such that for every $w \in C^{g}$ either $\vartheta(J(P)+w)$ vanishes identically on $X$ or it has exactly $g$ zeros $Q_{1}, Q_{2}, \ldots, Q_{g}$ (not necessarily distinct) with

$$
\sum_{i=1}^{g} J\left(Q_{i}\right)=-w+\Delta \quad \bmod L .
$$

In the following, we will use as vector $w$ certain values which will depend on our points $P_{1}, P_{2}, \ldots, P_{k}$. If we choose them generic the first case will never occur [12, theorem VI.3.3] . Immediately from (9) it follows that

$$
\vartheta\left(J(P)-g J\left(P_{k}\right)+\Delta\right)
$$

has a zero of order $g$ at $P_{k}$ and vanishes nowhere else.

The next building block is the prime form $E(P, R)[10, \bar{I}, \mathrm{p} .3 .210]$. It is a multi valued form on $X \times X$ of weight $-(1 / 2)$ in each argument ${ }^{1}$. It has the following properties:

$$
E(P, R)=0 \text { if and only if } P=R
$$

and this zero is of order 1 . It is antisymmetric in its arguments.

If $P$ is moved around the homology cycles

$$
P \mapsto P^{\prime}=P+\sum_{i=1}^{g} n_{i} a_{i}+\sum_{i=1}^{g} m_{i} b_{i}\left("=P^{\prime \prime}\right)
$$

we get (with ${ }^{t} m=\left(m_{1}, m_{2}, \ldots, m_{g}\right)$ )

$$
E\left(P^{\prime}, R\right)=\epsilon \cdot \exp \left(-\pi \mathbf{I}^{t} m \cdot \Pi \cdot m+2 \pi^{t} m \cdot(J(R)-J(P)) \cdot E(P, R) .\right.
$$

$\epsilon$ is a sign factor which depends on the cycle and the characteristic of the theta function used to défine the prime form.

The third building block is the $\sigma$ - differential. We define it as

$$
\sigma(P)=\theta\left(J(P)-g J\left(P_{k}\right)+\Delta\right) \cdot E\left(P, P_{k}\right)^{-g}
$$

It is a holomorphic multi valued form of weight $g / 2$ without zeros. Under (12) it transforms as

$$
\sigma\left(P^{\prime}\right)=\epsilon^{g} \cdot \exp \left(\mathbf{i} \pi(g-1)^{t} m \cdot \Pi \cdot m-\mathbf{i} 2 \pi^{t}(\Delta-(g-1) J(P))\right) \sigma(P) .
$$

If $\sigma^{\prime}$ is another $g / 2$ form with the same transformation (15) it will be a constant multiple of $\sigma$. Hence up to multiplication with a constant our $\sigma$ agrees with the $\sigma$ in $[11, p .31]$ and $[6]$.

IOf course, it can also be considered as single valued form on some covering $\bar{X} \times \bar{X}$, or as some section of a suitable line bundle on $\boldsymbol{X} \times \boldsymbol{X}$. 
Proposition. Let $g \geq 2$ and $\lambda \neq 0,1$ and

$$
n_{1}, n_{2}, \ldots, n_{k} \in \mathbf{Z}, \quad \sum_{i=1}^{k} n_{i}=M(\lambda)=(2 \lambda-1)(g-1)-1
$$

then there exits a constant $K \in \mathbb{C}$ such that

$$
\begin{gathered}
f^{\lambda}\left(n_{1}, n_{2}, \ldots, n_{k}\right)(P)=K \cdot \prod_{i=1}^{k-1} E\left(P, P_{i}\right)^{n_{1}} \cdot E\left(P, P_{k}\right)^{n_{k}-(2 \lambda-1) g} . \\
\vartheta\left(J(P)-g J\left(P_{k}\right)+\Delta\right)^{(2 \lambda-1)} \cdot \vartheta\left(J(P)+\sum_{i=1}^{k} n_{i} J\left(P_{i}\right)-(2 \lambda-1) \Delta\right) .
\end{gathered}
$$

PROOF: By an easy calculation (details in [4]) it is verified that the right hand side is a form of weight $\lambda$ which is single valued. It has exactly the zero order $n_{i}$ at the point $P_{i}$ (resp. pole order $-n_{i}$ at $P_{i}$ ). This is due to the fact that the last theta function term cannot increase the order of the zeros at the points $P_{i}$. Otherwise there would be a meromorphic section of $K^{\lambda}$ which has more zeros at the $P_{i}$ as the prescribed one. By a Riemann-Roch type argument it was proven in $[4]$, resp. $[8,(15)]$ that there is no such section beside the trivial one ${ }^{1}$. Because the generator on the left hand side is fixed up to a multiplicative constant this shows the claim.

The constant $K$ depends on the points $P_{i}$, the multiplicities $n_{i}$ and the weight $\lambda$. It can be calculated by calculating the lowest coefficient of the Laurent series expansion of the right hand side of (17) with respect to the coordinate $z_{k}$ around $P_{k}$ which was chosen to fix $f^{\lambda}\left(n_{1}, n_{2}, \ldots, n_{k}\right)$ (see $[6]$ in the case $k=2$ ).

Using the definition of $\sigma$ we can simply rewrite (17) as

$$
\begin{gathered}
f^{\lambda}\left(n_{1}, n_{2}, \ldots, n_{k}\right)(P)=K \cdot \prod_{i=1}^{k} E\left(P, P_{i}\right)^{n_{i}} \cdot \sigma(P)^{(2 \lambda-1)} . \\
\vartheta\left(J(P)+\sum_{i=1}^{k} n_{i} J\left(P_{i}\right)-(2 \lambda-1) \Delta\right)
\end{gathered}
$$

which reduces in the case $k=2$ to the form given in [6].

\footnotetext{
The nonvanishing of the last term can also be proven by using Riemann's theorem.
} 


\section{The construction via theta functions and prime forms in the case}

\section{$g \geq 1$. Exceptional cases.}

We consider first $g \geq 2$ and $\lambda=1$, hence $M(\lambda)=g-2$. As it was described in $[3,(22)-(24)]$ we have to consider 3 different types of generators. Type $[\beta,(23)]$ is the generic one

(19) $f^{\lambda}\left(n_{1}, n_{2}, \ldots, n_{k}\right), \quad$ at least one $n_{i} \leq-2$, all $n_{i} \neq-1, \sum_{i=1}^{k} n_{i}=M(\lambda)=g-2$.

Here again (17) and (18) give the generators. For example (17) specializes to

$$
\begin{gathered}
f^{1}\left(n_{1}, n_{2}, \ldots, n_{k}\right)(P)=K \cdot \prod_{i=1}^{k-1} E\left(P, P_{i}\right)^{n_{i}} \cdot E\left(P, P_{k}\right)^{n_{k}-g} . \\
\vartheta\left(J(P)-g J\left(P_{k}\right)+\Delta\right) \cdot \vartheta\left(J(P)+\sum_{i=1}^{k} n_{i} J\left(P_{i}\right)-\Delta\right) .
\end{gathered}
$$

To get the generators of the form $\mid s,(24)]$ we start for every pair of points $P_{i}, P_{j}, i<j$ with

$$
\omega_{i, j}:=d\left(\log \frac{E\left(P, P_{i}\right)}{E\left(P, P_{j}\right)}\right)
$$

It is a meromorphic (1-) differential on $X$, which is holomorphic on $X \backslash\left\{P_{1}, P_{2}\right\}$. It has pole order 1 at the points $P_{i}$ and $P_{j}$ with residues +1 and -1 . If integrated along the $a_{i}$ cycles it has zero periods $[10,11,3.212]$. By adding a suitable linear combination of holomorphic differentials $\omega_{l}$ we can achieve that the resulting differential has pure imaginary, periods $[9, p .116]$. The result is

$$
\begin{aligned}
& f^{1}(0, \ldots,-1,0, \ldots,-1, \ldots, 0)=\omega_{i, j}+ \\
& \quad \text { i } \sum_{r}\left(\sum_{s}\left((\operatorname{Im} \Pi)^{-1}\right)_{r \cdot} \cdot\left(\text { Re } \oint_{b_{0}} \omega_{i, j}\right)\right) \omega_{r} .
\end{aligned}
$$

Here $i$ and $j$ denote the indices $l$ with $n_{l}=-1$.

It remains $[\mathbf{3},(22)]$

$$
f^{1}\left(n_{1}, n_{2}, \ldots, n_{k}\right), \quad \text { all } n_{i} \geq 0, \sum_{i=1}^{k} n_{i}=(g-1) .
$$

To construct such a form we have to take an additional point $R \in X$ different from all $P_{i}$ and get 


$$
f^{1}\left(n_{1}, n_{2}, \ldots, n_{k}\right)(P)=K \cdot \prod_{i=1}^{k-1} E\left(P, P_{i}\right)^{n_{i}} \cdot E\left(P, P_{k}\right)^{n_{2}-g} \cdot E(P, R)^{-1}
$$

$$
\vartheta\left(J(P)-g J\left(P_{k}\right)+\Delta\right) \cdot \vartheta\left(J(P)+\sum_{i=1}^{k} n_{i} J\left(P_{i}\right)-J(R)-\Delta\right) .
$$

As in the general case this is a differential on $X$. The pole of $E(P, R)^{-1}$ at $P=R$ is annihilated by a zero of the last theta function term. Otherwise, the right hand side would be a differential with exactly one pole of order one. But this is impossible due to the residue theorem. The form does not depend on the point $R$ chosen, only the constant $K$ does. A possible choice could be the base point $Q$ of the Jacobi map.

Here again we can rewrite (24) as

$$
\begin{gathered}
f^{1}\left(n_{1}, n_{2}, \ldots, n_{k}\right)(P)=K \cdot \prod_{i=1}^{k} E\left(P, P_{i}\right)^{n_{4}} \cdot \sigma(P) \cdot E(P, R)^{-1} . \\
\vartheta\left(J(P)+\sum_{i=1}^{k} n_{i} J\left(P_{i}\right)-J(R)-\Delta\right) .
\end{gathered}
$$

This concludes the case $\lambda=1$.

Now we consider $g \geq 2, \lambda=0$ or $g=1$ and all $\lambda$. We have $M(0)=-g$. Again there are 3 types of generators as described in $[3,(28) \cdot(30)]$. Clearly, type $[3,(30)]$ is

$$
f^{0}(0, \ldots, 0) \equiv 1 \text {. }
$$

Type $[3,(28)]$ is the generic one

$$
f^{0}\left(n_{1}, n_{2}, \ldots, n_{k}\right), \quad \text { at least one } n_{i}>0, \sum_{i=1}^{k} n_{i}=-g .
$$

We get

$$
f^{0}\left(n_{1}, n_{2}, \ldots, n_{k}\right)(P)=K \cdot \prod_{i=1}^{k-1} E\left(P, P_{i}\right)^{n_{i}} \cdot E\left(P, P_{k}\right)^{n_{z}+g}
$$

$$
\vartheta\left(J(P)-g J\left(P_{k}\right)+\Delta\right)^{-1} \cdot \vartheta\left(J(P)+\sum_{i=1}^{k} n_{i} J\left(P_{i}\right)+\Delta\right)
$$


(29)

$$
\begin{gathered}
f^{0}\left(n_{1}, n_{2}, \ldots, n_{k}\right)(P)=K \cdot \prod_{i=1}^{k} E\left(P, P_{i}\right)^{n_{1}} \cdot \sigma(P)^{-1} \\
\vartheta\left(J(P)+\sum_{i=1}^{k} n_{i} J\left(P_{i}\right)+\Delta\right) .
\end{gathered}
$$

It remains the case $[\boldsymbol{s},(29)]$

$$
f^{0}\left(n_{1}, n_{2}, \ldots, n_{k}\right), \quad \text { all } n_{i} \leq 0, \sum_{i=1}^{k} n_{i}=-(g+1) .
$$

Again we choose a point $R$ and set

$$
\begin{gathered}
f^{0}\left(n_{1}, n_{2}, \ldots, n_{k}\right)(P)=K \cdot \prod_{i=1}^{k-1} E\left(P, P_{i}\right)^{n_{i}} \cdot E\left(P, P_{k}\right)^{n_{k}+g} \cdot E(P, R) \\
\vartheta\left(J(P)-g J\left(P_{k}\right)+\Delta\right)^{-1} \cdot \vartheta\left(J(P)+\sum_{i=1}^{k} n_{i} J\left(P_{i}\right)+J(R)+\Delta\right)
\end{gathered}
$$

or

$$
\begin{gathered}
f^{0}\left(n_{1}, n_{2}, \ldots, n_{k}\right)(P)=K \cdot \prod_{i=1}^{k} E\left(P, P_{i}\right)^{n_{1}} \cdot \sigma(P)^{-1} \cdot E(P, R) \\
\vartheta\left(J(P)+\sum_{i=1}^{k} n_{i} J\left(P_{i}\right)+J(R)+\Delta\right)
\end{gathered}
$$

The function has a zero at the point $R$. By varying $R$ we get different functions. This reflects the fact that in this case we were only able to fix the generator up to addition of a constant function and multiplication with a constant. The function can be fixed by requiring the duality relations given in [s,prop. 8$]$ to be valid. 


\section{The construction via the Welerstrab $\sigma$-function in the $g=1$ case.}

As it was done in $[1,13]$ for $k=2$ we will describe a different construction in the genus 1 case. Let $T$ be a torus (i.e. a genus 1 Riemann surface) given as $C / L$ with $L$ a lattice in $\mathbb{C}$. We can restrict ourselves to the case

$$
L=\mathbf{z} \oplus \tau \cdot \mathbf{z}, \quad \tau \in \mathbb{C}, \operatorname{Im} \tau>\mathbf{0}
$$

An arbitrary torus is always complex analytic isomorphic to such a torus. Weierstrab $\sigma$.function is defined as

$$
\sigma(z):=z \cdot \prod_{w \in L}\left(\left(1-\frac{z}{w}\right) \exp \left(\frac{z}{w}+\frac{1}{2}\left(\frac{z}{w}\right)^{2}\right)\right) .
$$

$\sigma$ is a holomorphic function. It is odd and has only zeros at the lattice points. The order of each zero is equal to one[14]. Of course, $\sigma$ is not a doubly periodic function, hence not a function on the torus. But if we take

$$
f(z)=\prod_{i=1}^{m}\left(\sigma\left(z-a_{i}\right)\right)^{n_{i}}
$$

we get that $f$ is a doubly periodic function with zeros of order $n_{i}$ at the points $a_{i}+L$ if and only if
(a) $\sum_{i=1}^{m} n_{i}=0$
(b) $\sum_{i=1}^{m} n_{i} a_{i}=0$.

Let $P_{1}, P_{2}, \ldots, P_{k}$ be the points chosen to define the Krichever-Novikov algebra on $T$. We choose $a_{i} \in \mathbb{C}$ with

$$
a_{i} \bmod L=P_{i}, \quad i=1, \ldots, k
$$

and fix them. Because the canonical bundle $K$ on $T$ is trivial we have

$$
f^{\lambda}\left(n_{1}, n_{3}, \ldots, n_{k}\right)=f^{0}\left(n_{1}, n_{2}, \ldots, n_{k}\right)(d z)^{\lambda}
$$

Hence it is enough to determine $f^{0}\left(n_{1}, n_{2}, \ldots, n_{k}\right)$.

Again we have the 3 different types of section 3 . (26) remains the same: $f^{0}(0, \ldots, 0) \equiv 1$. In the generic situation (27) we set

$$
b=-\left(\sum_{i=1}^{k} n_{i} a_{i}\right)
$$


and get

$$
f^{0}\left(n_{1}, n_{2}, \ldots, n_{k}\right)=C \cdot \prod_{i=1}^{k}\left(\sigma\left(z-a_{i}\right)\right)^{n_{i}} \cdot \sigma(z-b) .
$$

The conditions (36) are fullfilled. Hence the right hand side of (40) is a doubly periodic function. If the $P_{i}$ are chosen to be in general position the point $b \bmod L$ will never coincide with any $a_{i} \bmod L$. Otherwise $\sigma(z-b)$ would increase the order of the zero at the point $P_{i}$. As in the generic case (section 2.), we know there is no such nontrivial function. Hence $(40)$ is in fact the generator we are looking for. If we choose the local coordinate $z_{k}=z-a_{k}$ around the point $P_{k}$ we can determine the constant $C$ as

$$
C^{-1}=\prod_{i=1}^{k-1}\left(\sigma\left(a_{k}-a_{i}\right)\right)^{n_{i}} \cdot \sigma\left(a_{k}-b\right)
$$

The remaining case (30) consists of two possibilities

$$
f^{0}(0, \ldots, 0,-2,0, \ldots, 0) \text { and } f^{0}(0, \ldots,-1,0, \ldots,-1,0, \ldots, 0) \text {. }
$$

Let the points of nonzero order be $a_{i}$, resp. $a_{i}$ and $a_{j}$ with $i<j$. We determine $w_{1}$ and $w_{2}$ such that

$$
w_{1}+w_{2}=-2 a_{i} \text { resp. } w_{1}+w_{2}=-\left(a_{i}+a_{j}\right)
$$

and that

$$
w_{1}, w_{2} \neq a_{l} \bmod L \text { for } l=1, \ldots, k \text {. }
$$

Generators of the types in (42) are now given by

$$
\begin{gathered}
f^{0}(0, \ldots, 0,-2,0, \ldots, 0)=C \sigma\left(z-a_{i}\right)^{-2} \cdot \sigma\left(z-w_{1}\right) \cdot \sigma\left(z-w_{2}\right)+D \\
f^{0}(0, \ldots,-1,0, \ldots,-1,0, \ldots, 0)=C \sigma\left(z-a_{i}\right)^{-1} \cdot \sigma\left(z-a_{j}\right)^{-1} \\
\sigma\left(z-w_{1}\right) \cdot \sigma\left(z-w_{2}\right)+D .
\end{gathered}
$$

Here $C$ and $D$ are constants which can be suitable fixed. In the case of the basis in [s] (where only (46) and only $j=k$ occur) the choice compatible with the duality is

$$
D=0 \text { and } C^{-1}=\sigma\left(a_{k}-a_{i}\right)^{-1} \cdot \sigma\left(a_{k}-w_{1}\right) \cdot \sigma\left(a_{k}-w_{2}\right) .
$$




\section{The construction via rational functions in the $g=0$ case.}

For the sake of completeness I will repeat the generators for the $g=0$ case $\left(X=\mathbb{P}^{1}\right)$. They have already appeared in $[3,5]$. We choose a parametrization $z$ of $\mathbb{P}^{1}$, such that $P_{1}$ corresponds to $z=0$ and $P_{k}$ corresponds to $z=\infty$. The remaining points $P_{l}$ correspond to $a_{1} \neq 0, \infty$. Let $n_{i}$ be given such that $\sum_{i=1}^{k} n_{i}=M(\lambda)=-2 \lambda$ then we have

$$
f^{\lambda}\left(n_{1}, n_{2}, \ldots, n_{k}\right)(z)=(-1)^{\lambda} \cdot z^{n_{2}} \prod_{i=2}^{k-1}\left(z-a_{i}\right)^{n_{1}}(d z)^{\lambda} .
$$
In fact, if we calculate this form in the local coordinate around $P_{k}$ given by $w=\frac{1}{z}$ we
get

$$
f^{\lambda}\left(n_{1}, n_{2}, \ldots, n_{k}\right)(z(w))=w^{\left(-\sum_{i=1}^{k-1} n_{i}-2 \lambda\right)} \prod_{i=2}^{k-1}\left(1-a_{i} w\right)^{n_{i}}(d w)^{\lambda}
$$

Hence we have exactly the prescribed zero of order $n_{k}=-2 \lambda-\sum_{i=1}^{k-1} n_{i}$ at $P_{k}$.

\section{Note added.}

While this manuscript was typed I received a written version of a talk presented by Reiner Dick at the workshop: Physics and Geometry 1989 [15] where he gave expressions similar to those in section 3 and 4 for his generators in [5]. 


\section{References}

1. I.M. Krichever and S.P. Novikov, Algebras of Virasoro Type, Riemann Surfaces and Structures of the Theory of Solitons, Funk. Anal. i. Pril. 21 (2) (1987), 46.

2. I.M. Krichever and S.P. Novikov, Virasoro Type Algebras, Riemann Surfaces and Strings in Minkoweki Space, Funk. Anal. i. Pril. 21 (4) (1987), 47.

3. M. Schlichenmaier, Krichever-Novikov Algebras for More Than Two Points, KA.THEP-1989.6 (April 89) and Man.Fak.M.I Mannheim 97-1989 (to appear in Lett.Math.Phys.).

4. M. Schlichenmaier, Verallgemeinerte Krichever-Novikov Algebren und deren Darstellungen, (to appear).

5. R. Dick, Krichever-Novikov-like Bases on Punctured Riemann Surfaces, Desy 89-059 (May 89) (to appear in Lett.Math.Phys.).

6. L. Bonora, A. Lugo, M. Matone and J. Russo, A Global Operator Formalism on Higher Genus Riemann Surfaces: b-c Systems, Comm. Math. Phys. 128 (1989), 329-352.

7. A. Lugo and J. Russo, Hamiltonian Formulation and Scattering Amplitudes in String Theory at Genus g, SISSA 83/88/EP.

8. L. Bonora, M. Matone and F. Toppan, Real Weight $b-c$ Systems and Conformal Field Theory in Higher Genue, ISAS/SISSA 55/89/EP.

9. M. Schlichenmaier, "An Introduction to Riemann Surfaces, Algebraic Curves and Moduli Spaces", Lecture Notes in Physics Vol. 322, Springer, 1989.

10. D. Mumford, “Tata Lectures on Theta I,II", Birkhäuser, 1983(1984).

11. J.D. Fay, "Theta Functions on Riemann Surfaces", Lecture Notes in Math. Vol.352, Springer, 1973.

12. H. M. Farkas, I. Kra, "Riemann Surfaces", Springer, 1980.

13. L. Mesincescu, R.I. Nepomechie, C.K. Zachos, (Super)conformal Algebra on the (Super)torus, UMTG-144 and ANL-Hep-Pr-88-23.

14. A. Hurwitz, R. Courant, "Allgemeine Funktionentheorie und elliptische Funktionen", Springer, 1964.

15. R. Dick, Holomorphic Differentials on Punctured Riemann Surfaces, Talk presented at the workshop : Physics and Geometry, Lake Tahoe 3.8 July 1989 (to appear in the proceedings). 